OPEN ACCESS

Edited by:

Min Yue,

Zhejiang University, China

Reviewed by:

Guijun Wang

Anhui Agricultural University, China

Hongjun Chen,

Chinese Academy of Agricultural Sciences (CAAS), China

Guoqiang Zhu,

Yangzhou University, China

*Correspondence:

Xinyu Zhang

zxy@yzu.edu.cn

Fang Wang

vetwangfang@126.com

Specialty section

This article was submitted to Veterinary Infectious Diseases,

a section of the journal

Frontiers in Veterinary Science

Received: 22 October 2020 Accepted: 23 December 2020

Published: 12 February 2021

Citation:

Zhang $X$, Yu S, Cheng D, Feng Y,

Yang $Y$, Sun $H$, Ding J and Wang $F$ (2021) An Attenuated Escherichia coli

K88ac LT(S63K) $\triangle S T$ tb Efficiently

Provides Protection Against Enterotoxigenic Escherichia coli in the Mouse Model.

Front. Vet. Sci. 7:620255. doi: 10.3389/fvets.2020.620255

\section{An Attenuated Escherichia coli K88ac LT(S63K) $\Delta S T b$ Efficiently Provides Protection Against Enterotoxigenic Escherichia coli in the Mouse Model}

\author{
Xinyu Zhang ${ }^{1,2 *}$, Shupei Yu ${ }^{1,2}$, Darong Cheng ${ }^{1,2}$, Yu Feng ${ }^{3}$, Yuefei Yang ${ }^{1,2}$, \\ Huaichang Sun ${ }^{1,2}$, Jiabo Ding $^{3}$ and Fang Wang ${ }^{3 *}$ \\ ${ }^{1}$ College of Veterinary Medicine, Yangzhou University, Yangzhou, China, ${ }^{2}$ Jiangsu Co-innovation Center for Prevention and \\ Control of Important Animal Infectious Diseases and Zoonoses, Yangzhou, China, ${ }^{3}$ Department of Biologics Detection \\ Technology, China Institute of Veterinary Drugs Control, Beijing, China
}

To develop an attenuated vaccine candidate against K88ac enterotoxigenic Escherichia coli (ETEC), a novel Escherichia coli (E. coli) K88ac $L T(S 63 K) \Delta S T b$ with $L T(S 63 K)$ mutation and ST1 deletion was generated using site mutagenesis and $\lambda$-Red homologous recombination based on wild paternal ETEC strain C83902. E. coli K88ac $L T(S 63 K) \Delta S T$ tb showed very similar fimbriae expression and growth kinetics to the wild strain C83902, but it was significantly attenuated according to the results of a rabbit ligated ileal loop assay and mouse infection study. Oral inoculation with E. coli K88ac $L T(S 63 K) \Delta S T b$ stimulated the mucosa immune response and induced the secretion of IgA to K88ac in the intestines in mice. A challenge experiment revealed that the attenuated strain provided efficient protection against C83902 in the following 7 days and at the 24th day post-inoculation, suggesting that the attenuated isolate could act as an ecological protectant and vaccine in preventing K88ac ETEC.

Keywords: ETEC, K88ac, LT(S63K), STb deletion, attenuation, protection

\section{INTRODUCTION}

Porcine neonatal diarrhea is a global problem in the pig industry (1). It is mainly caused by enterotoxigenic Escherichia coli (ETEC) strains, which may express fimbriae K88 (F4) or F18 related to bacterial colonization in the intestine. The secreted enterotoxins, including heat labile toxin $(L T)$, heat-stable toxin type I (STa), heat-stable toxin type II (STb), and Shigella toxin type II (Stx2e) $(2,3)$, are the direct causes of diarrhea. They enhance the capillary permeability and increase the secretion of intestinal gland cells so that a large amount of liquid is accumulated in the small intestine, far exceeding the absorption capacity of the small intestine and leading to diarrhea. The loss of water, sodium, and carbonate ions results in dehydration, metabolic acidosis, and hyperkalemia, and it may induce heart failure and even death (4).

In order to prevent piglet diarrhea caused by ETEC, pregnant sows are usually immunized with live non-enterotoxigenic E. coli or protective antigens such as recombinant antigens of fimbriae and enterotoxins. Newborn piglets obtain passive protective antibodies through the colostrum $(5,6)$. Some breeds of sows cannot express receptors related to the adhesion of fimbriae, such as K88 receptor-negative sows, which do not produce or transfer specific antibodies against K88 in colostrum even post-immunization. These heterozygous 
piglets cannot obtain effective passive protection (1). The passive protection obtained through nursing does not effectively prevent post-weaning diarrhea. Egg yolk antibodies against adhesion fimbriae and enterotoxins might play a role in this period (7). In addition, some probiotics, such as Bacillus subtilis, Bacillus licheniformis, Clostridium butyricum, and yeasts, are administered to piglets. These probiotics use the nutrients in the intestinal tract for reproduction (8). During reproduction, lactic acid, butyric acid, antimicrobial peptides, and other substances are produced, causing the $\mathrm{pH}$ of the intestinal environment to decrease and inhibiting the growth of harmful bacteria. Probiotics interact with harmful bacteria such as Escherichia coli, Salmonella, and Clostridium welchii to compete for space and nutrition (9). Recently, a recombinant Lactobacillus expressing K88 and K99 fimbria was used to immunize mice orally. Among the immunized mice, $85 \%$ resisted a wild ETEC strain (10), indicating that this may be a useful new strategy in controlling piglet diarrhea.

The $L T$ and $S T b$ genes are located in a large plasmid in ETEC K88ac, a dominant ETEC expressing K88 fimbria that is related to diarrhea $(11,12)$. $L T$ plays a role in assisting bacterial adhesion and acts as a good adjuvant for mucosal immunity. Research has indicated that it might significantly enhance the responses of the humoral immune system and induce a high level of antibodies against the co-immune antigens in the intestines and serum, while at the same time avoiding immune tolerance and triggering a long-term memory effect to those co-immune antigens $(13,14)$. The subunit A of LT contains a domain with ADP-ribosylating activity. Subunit A can bind to NAD, and the serine of amino acid 63 of subunit $A$ is located at the bottom of the NAD-binding chamber, which is closely related to virulence (15). Mutating serine (63) to lysine might cause the virulence of recombinant protein $\mathrm{LT}$ to be greatly reduced while retaining its strong adjuvanticity (16).

Our purpose was to construct an oral whole-cell vaccine against K88ac ETEC. In this paper, we developed an attenuated E. coli by mutating nucleotides $187-189$ of $L T$ from TCT (serine) to AAA (lysine) and deleting $S T b$ using $\lambda$-Red homologous recombination. This isolate could efficiently protect vaccinated mice against wild ETEC.

\section{MATERIALS AND METHODS}

\section{Animals, Bacterial Strains, Plasmids, and Primers}

Eight-week-old female Balb/c mice and Japanese big-ear white rabbits with body weights between 2 and $3 \mathrm{~kg}$ were purchased from the Institute of Comparative Medicine, Yangzhou University (Jiangsu, China). Animals were raised under a standard 12-h light/dark cycle, in conventional housing conditions at $20 \pm 1^{\circ} \mathrm{C}$ and $50 \pm 10 \%$ relative humidity. All of the experimental animal operating procedures were in line with the guidelines for the management and use of experimental animals (2016 edition) and approved by the Animal Care and Use Committee of Yangzhou University. K88ac ETEC strain $\mathrm{C} 83902\left(\mathrm{LT}^{+}, \mathrm{STb}^{+}\right)$, used to construct the attenuated E. coli $\mathrm{K} 88 \mathrm{ac}$, was from the China Institute of Veterinary Drug Control
(Beijing, China). DH5 $\alpha$ competent cells and pUC18 plasmid were purchased from Takara Biotechnology (Dalian) Co., Ltd. Plasmids pSG76-CS and pSTKST as reported (17) were kindly provided by Prof. Lijun Zhang, Shenyang Agricultural University (Liaoning, China). Monoclonal antibody against a factor of K88 fimbriae was kindly provided by Prof. Song Gao, Yangzhou University. The primers (Table 1) used in this paper were designed according to the plasmid pUMNK88 (GenBank accession No. CP002730) and synthesized by Sangon Biotech (Shanghai) Co., Ltd., China.

\section{Construction of Mutated E. coli K88ac LT(S63K) $\Delta S T b$ From C83902}

Deletion of $S T b$ was performed using an E. coli gene knockout kit (Jiangsu RuiYang BioTech Co., Ltd., China) as protocol and the construction process as shown in Figure 1A. Briefly, PCR fragments using primers $\mathrm{P} 1 / \mathrm{P} 2$ and $\mathrm{P} 3 / \mathrm{P} 4$ amplified from the C83902 strain were cloned into restriction endonuclease sites EcoR I/Sma I and Sma I/Hind III of plasmid pUC18, then the dif-Gm-dif DNA digested with Sma I was inserted into the restriction endonuclease Sma I site. The constructed plasmid above was used as the template, and the recombinant cassette containing the left homologous arm, right homologous arm, and dif-Gm-dif amplified by PCR using primers P5/P6 was transformed into C83902 with plasmid pKD46 induced by Larabinose at a final concentration of $4 \mu \mathrm{g} / \mathrm{ml}$. After homologous recombination, the gentamicin resistance gene $\left(\mathrm{Gm}^{r}\right)$ was deleted by the dif/Xer recombinant system of the E. coli, and a 34-bp sized dif sequence was left in the large plasmid of the E. coli named K88ac ETEC $\Delta S T b$, which was verified by PCR using primers $\mathrm{P} 7 / \mathrm{P} 8$.

TABLE 1 | Primers used in constructing E. coli K88ac $L T(S 63 K) \triangle S T b$.

\begin{tabular}{|c|c|c|}
\hline Primer & Sequence $\left(5^{\prime}>3^{\prime}\right)$ & Size (bp) \\
\hline P1 & ctgaattcCGCTGCATTATTGATITAGGAC & 378 \\
\hline P2 & cgcccgggCACCTCAACCTTATTGCTATAAG & \\
\hline P3 & cgcccgggTATATTTATCAATAGCATTCAGCACC & 356 \\
\hline P4 & gcgaagctt CCGGATGATCTCTAAATATGAAT & \\
\hline P5 & CATATAAAAGCCCACTGG & 1034 \\
\hline P6 & CПIITATGAAAAATTATIITG & \\
\hline P7 & ATTGTTGACATGAACAGC & 800 \\
\hline P8 & GCGATCTCCTTCGTTAGG & \\
\hline P9 & $\begin{array}{l}\text { gaggaacacaaaccggetttgtcagatat } \\
\text { gatgacggatatgtttccactAAActtagtttgagaagtgctcac }\end{array}$ & 1390 \\
\hline P10 & $\begin{array}{l}\text { cgagctcggtacccggggatccgcTAGGGA } \\
\text { TAACAGGGTAATgtcctgctaagtgagcactt } \\
\text { ctcaaactaagTTag }\end{array}$ & \\
\hline P11 & $\begin{array}{l}\text { gaatatcctgataatatagactgtcctgct } \\
\text { aagtgagcacttctcaaactaagATTACCCTGT } \\
\text { TATCCCTAtagcggccgcaaaaattaaaaatgaag }\end{array}$ & \\
\hline P12 & GAGGAACACAAACCGGCTITG & 106 \\
\hline P13 & GAATATCCTGATAATATAGACTG & \\
\hline P14 & TAGAGACCGGTATTACAGAAATCTGA & 282 \\
\hline P15 & TCATCCCGAATTCTGTTATATATGTC & \\
\hline
\end{tabular}




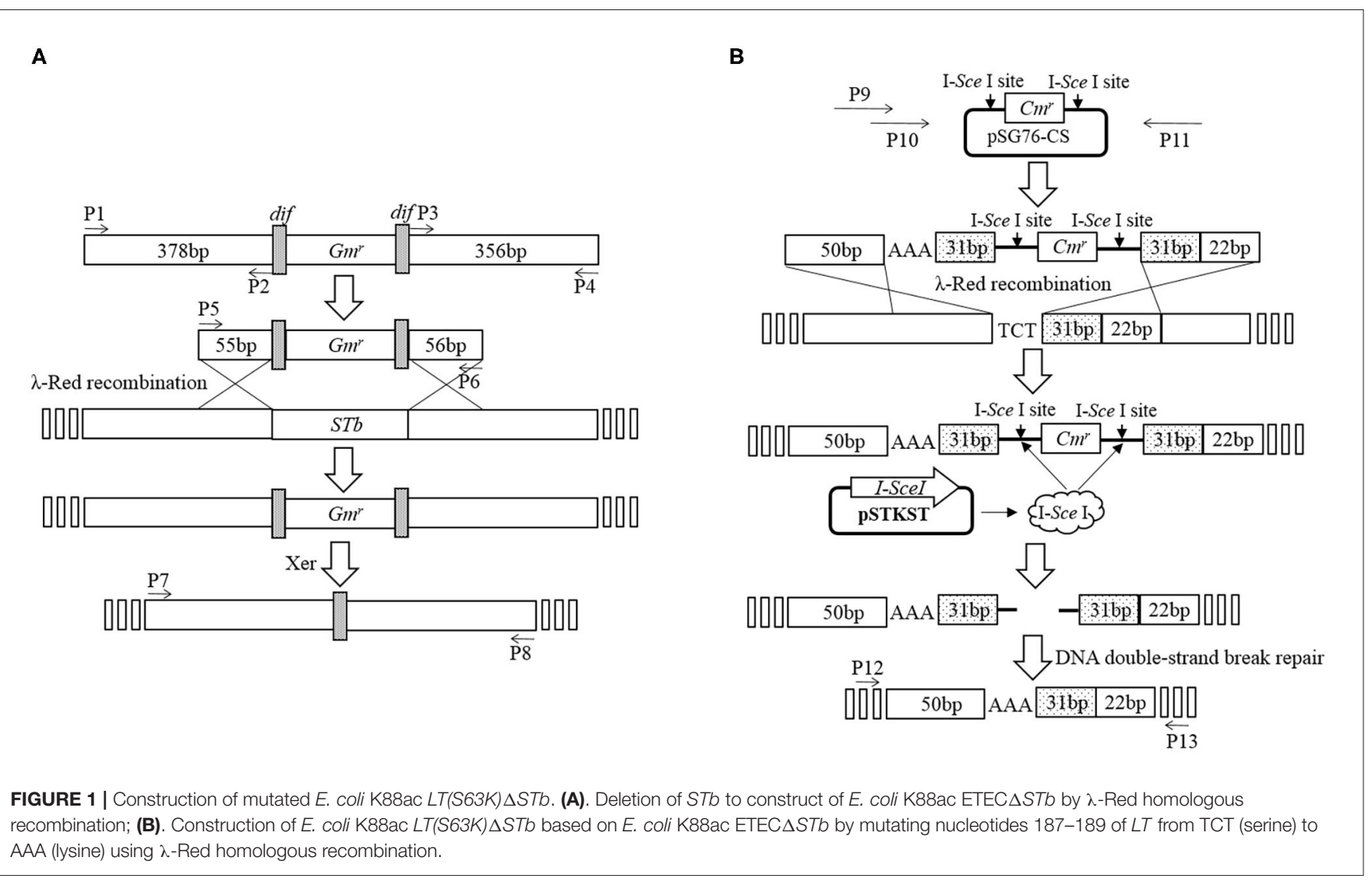

Using plasmid pSG76-CS as template, another recombinant cassette containing left and right homologous arms, mutated nucleotides, and chloramphenicol resistance gene $\left(\mathrm{Cm}^{r}\right)$ with two endonuclease I-SceI sites on two sides was amplified by overlapping PCR using primers P9/P10/P11. The PCR product was transformed into K88ac ETEC $\Delta S T 1$ with plasmid pKD46 induced by L-arabinose to insert the amplicon into $L T$ by $\lambda$-Red recombination. Then, plasmid pKD46 was removed at a temperature of $40^{\circ} \mathrm{C}$ and the plasmid pSTKST was transformed into the recombinant $E$. coli. After being induced by chlorotetracycline (Shanghai Yuanmu BioTech Co., Ltd., China) at a final concentration of $30 \mu \mathrm{g} / \mathrm{ml}$, endonuclease ISceI expressed by plasmid pSTKST was used to delete the $\mathrm{Cm}^{r}$ gene. The large plasmid in E. coli was repaired by the method of broken-end recombination, and the mutation was fixed in $L T$. Then, plasmid pSTKST was removed at $40^{\circ} \mathrm{C}$ and the recombinant $E$. coli named K88ac $L T(S 63 K) \Delta S T b$ was constructed and verified by sequencing the PCR product amplified using primers P12/P13 as Figure 1B.

\section{Indirect Immunofluorescence Assay}

E. coli strains including K88ac $L T(S 63 K) \Delta S T$, C83902, and DH5 $\alpha$ were inoculated in TSB liquid medium and grown for $36 \mathrm{~h}$. One $\mathrm{ml}$ of the bacterial solutions was centrifuged at $8,000 \mathrm{~g} / \mathrm{min}$ for $5 \mathrm{~min}$, washed gently with phosphate buffered saline (PBS) using a wide-bore tip, and resuspended in $1 \mathrm{ml}$ PBS. Five $\mu \mathrm{L}$ of the suspensions was coated on glass slides and immobilized using the heat fixing method. The fixed bacteria were blocked with
$5 \%$ goat serum for $15 \mathrm{~min}$ at room temperature and rinsed with PBS. Then, the slides were incubated with murine monoclonal antibody against a factor of $\mathrm{K} 88$ fimbriae diluted in 5\% goat serum for $45 \mathrm{~min}$. After being washed with PBS 3 times, the bacteria were incubated with 1:10,000 dilution of goat anti-mouse IgG conjugated with FITC (Abcam, UK) for another $45 \mathrm{~min}$. The glass slides were washed in PBS 3 times, and the dyed bacteria were observed under the oil immersion lens of the microscope.

\section{Growth Characteristics}

E. coli $\mathrm{K} 88 \mathrm{ac} L T(S 63 K) \Delta S T b$ and $\mathrm{C} 83902$ were inoculated into liquid LB medium $(\mathrm{pH}=7.2)$ and cultured overnight at $37^{\circ} \mathrm{C}$. After the concentration was adjusted to OD600 $=0.04$ with a UV spectrophotometer, the bacterial suspensions were transferred into LB medium at ratio of $1: 100$ and cultured at $37^{\circ} \mathrm{C}$ with shaking at $220 \mathrm{rpm}$. At the time points of $1.5 \mathrm{~h}, 3 \mathrm{~h}, 4 \mathrm{~h}, 6 \mathrm{~h}$, $8 \mathrm{~h}, 10 \mathrm{~h}, 12 \mathrm{~h}, 14 \mathrm{~h}$, and $16 \mathrm{~h}$ post-inoculation, the OD600 values of the suspensions were measured and the growth rate of the attenuated strain was compared with that of wild bacterium.

\section{In vivo Rabbit Ligated lleal Loop Assay}

E. coli bacteria including C83902, K88ac $L T(S 63 K) \Delta S T$, and DH5 $\alpha$ were grown in an LB medium with horizontal shaking at $37^{\circ} \mathrm{C}$ overnight, then transferred into a 10-ml CAYE medium at a ratio of 1:100 and cultured at $37^{\circ} \mathrm{C}$. Seventy-two hours later, 2,640 units of polymyxin B were added and cultured for an extra $0.5 \mathrm{~h}$. The bacterial suspensions were centrifuged at $10,000 \times g$ 
for $10 \mathrm{~min}$, and the obtained cell-free culture supernatants were filtered with sterile $0.22 \mu \mathrm{m}$-pore filters (Millipore, France).

The rabbit ligated ileal loop assay was performed as described with slight modifications (18). In detail, two Japanese big-ear white rabbits were fasted for $24 \mathrm{~h}$ and then anesthetized by the intramuscular injection of xylazine hydrochloride (SuMianXin; Tat Animal Pharmaceutical Co., Ltd. of Jilin Province, China) and zolazepam chloridrate (Zoletil; Virbac Sante Animal, France) at a dose of $0.08 \mathrm{ml} / \mathrm{kg}$ and $0.04 \mathrm{ml} / \mathrm{kg}$, respectively. Using laparotomy, the empty mid-ileums were exposed. Ileal loops at lengths of $5 \mathrm{~cm}$ were ligated at intervals of $3 \mathrm{~cm}$. Inoculums of $1 \mathrm{ml}$ of cell-free culture supernatants or sterile CAYE medium as control were inoculated into each ligated loop of a rabbit using a 25-gauge needle, and repeated in another rabbit. After the abdominal cavities were closed, the rabbits were kept for $12 \mathrm{~h}$, then euthanized by carbon dioxide inhalation. The volume of secretion in each ligated loop was measured, and the average ratio (the volume of liquid to the length of the intestine) of each sample was calculated. The average ratio was higher than 1.0, indicating that the enterotoxin existed in cell-free culture supernatant.

\section{Infection Study in vivo}

The plasmid pUC18 was transformed into E. coli K88ac $L T(S 63 K) \Delta S T b$ and $\mathrm{C} 83902$, and the two recombinant bacteria were reproduced in TSB medium with $1 \mu \mathrm{g} / \mathrm{ml}$ ampicillin. Forty-eight hours before the inoculation of bacteria, fructose and ampicillin were added to the animals' drinking water at concentrations of $67 \mu \mathrm{g} / \mathrm{ml}$ and $1 \mu \mathrm{g} / \mathrm{ml}$ respectively to encourage the mice to drink and get rid of the other bacteria; $12 \mathrm{~h}$ before inoculation, the mice were fasted; and $4 \mathrm{~h}$ before inoculation, the drinking water was changed into sterile water and all of mice were injected with cimetidine $(50 \mathrm{mg} / \mathrm{kg}$ ) intraperitoneally. The mice were divided into three groups and raised in independent units. According to the preliminary experimental results, each mouse of group $1(n=10)$, group 2 ( $n$ $=10)$, and group $3(n=10)$ was inoculated $0.4 \mathrm{ml}$ (approximately $10^{9} \mathrm{CFU}$ ) of K88ac ETEC (C83902), K88ac LT(S63K) $\Delta S T b$, and sterile PBS by intragastric administration, respectively. Clinical symptoms were observed in the following 7 days, and clinical signs were recorded and scored every day as described with slight modifications (19): 0, healthy; 1, mental depression and ruffled fur; 2, hunchbacked appearance, shivering, and dark urine; 3, mild diarrhea; 4, severe diarrhea; 5, moribund or dead. The feces were collected over the following 33 days for detecting the inoculated E. coli and IgA secreted in the intestinal tract.

\section{E. coli K88ac $L T(S 63 K) \Delta S T b$ and Wild Strain Detection}

Feces collected were inoculated into the TSB liquid medium with $1 \mu \mathrm{g} / \mathrm{ml}$ ampicillin and shaken in an orbital incubator at a speed of $220 \mathrm{rpm}$ for $16 \mathrm{~h}$. PCR assay was performed to detect $L T$ or mutant $L T$ genes in wild or attenuated strains using primers P14 and P15 with an initial denaturation at $94^{\circ} \mathrm{C}$ for $5 \mathrm{~min}$, followed by 30 cycles of denaturation at $94^{\circ} \mathrm{C}$ for $30 \mathrm{~s}$, annealing at $54^{\circ} \mathrm{C}$ for $30 \mathrm{~s}$, extension at $72^{\circ} \mathrm{C}$ for $1 \mathrm{~min}$, and an extra extension at $72^{\circ} \mathrm{C}$ for $5 \mathrm{~min}$. PCR products were visualized in
$1.5 \%$ agarose gel stained with ethidium bromide using the gel image documentation system.

\section{IgA Detection by Indirect ELISA}

The K88ac fimbriae of C83902 were isolated and purified as described (6); the purified fimbriae were adjusted to a concentration of $10 \mathrm{ng} / \mathrm{ml}$ in $0.05 \mathrm{~mol} / \mathrm{l}$ carbonate buffered saline at a $\mathrm{pH}$ of 9.6. Then, $100 \mu \mathrm{l}$ of the diluted antigen was coated into the wells of the 96-well assay plates (Corning, America) overnight at $4{ }^{\circ} \mathrm{C}$. The plates were washed twice with PBS containing $0.05 \%$ Tween 20 (PBST) and blocked with $200 \mu \mathrm{l} /$ well of PBST with $5 \%$ non-fat dry milk for $1 \mathrm{~h}$ at $37^{\circ} \mathrm{C}$. The plates were then washed twice more with PBST. Subsequently, $0.5 \mathrm{ml}$ PBS was added into $0.1 \mathrm{~g}$ mice feces collected at intervals of 3 days, mixed completely, and centrifuged at 12,000 rpm for $5 \mathrm{~min}$. The supernatants were collected and transferred into 96-well plates at $100 \mu \mathrm{l} /$ well, incubated for $1 \mathrm{~h}$ at $37^{\circ} \mathrm{C}$, and washed three times with PBST. A 1:5,000 dilution of HRP-conjugated goat anti-mouse IgA antibody (Abcam, UK) in a blocking solution was dispensed into the plates at $100 \mu \mathrm{l} /$ well and incubated for $1 \mathrm{~h}$ at $37^{\circ} \mathrm{C}$. The plates were washed again with PBST, then $100 \mu \mathrm{l} /$ well of 3,3,5,5-tetramethylbenzidine substrate (Sangon Biotech, China) was added, and the reaction was stopped with $50 \mu \mathrm{l} /$ well of $2 \mathrm{~mol} / \mathrm{L}$ sulfuric acid solution $15 \mathrm{~min}$ later. The antibody level was measured by the microplate reader (Biotech, America) at a wavelength of $450 \mathrm{~nm}$.

\section{Protection Assay}

Fifty mice were divided into three groups (five mice each in Group 1 and Group 2 as control groups and 40 mice in Group 3 as the experimental group), and mice in all of the groups were treated with fructose, antibiotic, and cimetidine as described above. Then, the mice in Group 1 took C83902 with plasmid pUC18 orally at a dose of $10^{9} \mathrm{CFU} / \mathrm{each}$, and mice in Group 2 and Group 3 took E. coli K88ac $L T(S 63 K) \Delta S T b$ with plasmid pUC18 orally at a dose of $10^{9} \mathrm{CFU} /$ each. Group 3 performed as the challenge group. Five mice in Group 3 were selected at random and raised in independent units, then treated with C83902 harboring plasmid pUC18 at a dose of $10^{9} \mathrm{CFU} /$ each at intervals of $24 \mathrm{~h}$ post-inoculation over the following 7 days. The last five mice were treated on the 24th day. Clinical signs were recorded over the 3 days following the treatment and scored as described above.

\section{Analysis}

Data were analyzed using the GraphPad Prism 5.00 software.

\section{RESULTS}

\section{Generation of $E$. coli K88ac $L T(S 63 K) \Delta S T b$}

To generate the mutant E. coli K88ac, two $\lambda$-Red recombinations were carried out. Firstly, the $\lambda$-Red recombination system was used to knock out the $S T b$ gene located on the large plasmid harbored in C83902. The constructed K88ac ETEC $\Delta S T b$ was verified by PCR and sequence analysis, and the results indicated that the $S T b$ gene was replaced by a 34-bp-sized dif (Figure 2A). Secondly, based on the procedure above, a markerless mutation 
was performed to change the serine at position amino acid 63 of heat-labile toxin into lysine using $\lambda$-Red recombination again. Sequencing results showed that the codon TCT(S) was mutated to a codon $\mathrm{AAA}(\mathrm{K})$ (Figure 2B), and E. coli $\mathrm{K} 88 \mathrm{ac}$ $L T(S 63 K) \Delta S T b$ was obtained.

\section{K88ac $L T(S 63 K) \Delta S T b$ Could Efficiently Express Fimbriae}

Usually, fimbriae of K88ac expressed on the surface of E. coli is encoded by the genes in the large plasmid, which is important in the adhesion of susceptible cells in intestinal mucosa and plays important roles in the pathogenic process. To avoid knocking out fimbriae genes by mistake, K88ac were detected by immunofluorescence assay. The results of IFA showed that there was bright fluorescence around the surfaces of K88ac $L T(S 63 K) \Delta S T b$ and C83902, but no fluorescence on the surface of control DH5 $\alpha$ as shown in Figure 3, indicating that the mutation and knockout of enterotoxin genes had no effect on the expression of K88ac fimbriae.

\section{K88ac LT(S63K) $\Delta S T b$ Showed Similar Growth Kinetics to the Wild Strain}

To ensure the growth character of E. coli K88ac LT(S63K) $\Delta S T b$, the fresh bacterial fluid of the attenuated Escherichia coli was inoculated into the LB medium at a ratio of 1:100 and cultured at $37^{\circ} \mathrm{C}$. C83902 was set as the control, and concentrations of bacterial fluids at different times post-inoculation were measured using the $\mathrm{OD}_{600}$ value obtained by a UV spectrophotometer. As shown in Figure 4, the growth rate of the attenuated strain was a little lower compared with that of the original bacterium in first several hours, but at $10-16 \mathrm{~h}$ post-inoculation the bacterial reproduction quantity of the two bacteria reached the same
A

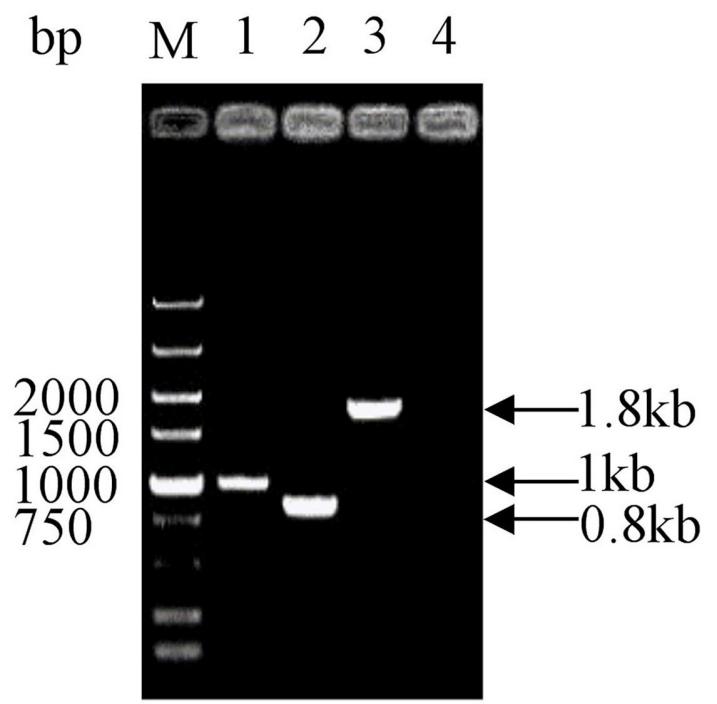

B

FIGURE 2 | Identification of mutated E. coli K88ac $L T(S 63 K) \Delta S T$. (A). Identification of E. coli K88ac ETEC $\triangle S T b$ by PCR, M. DNA marker, 1. Wild strain C83902, 2. E. coli K88ac ETEC $\Delta S T$, 3. E. coli K88ac ETEC $\Delta S T$ b before removal of gentamicin resistance gene, 4. Negative control; (B). Sequence result of mutating TCT (serine) to AAA (lysine).
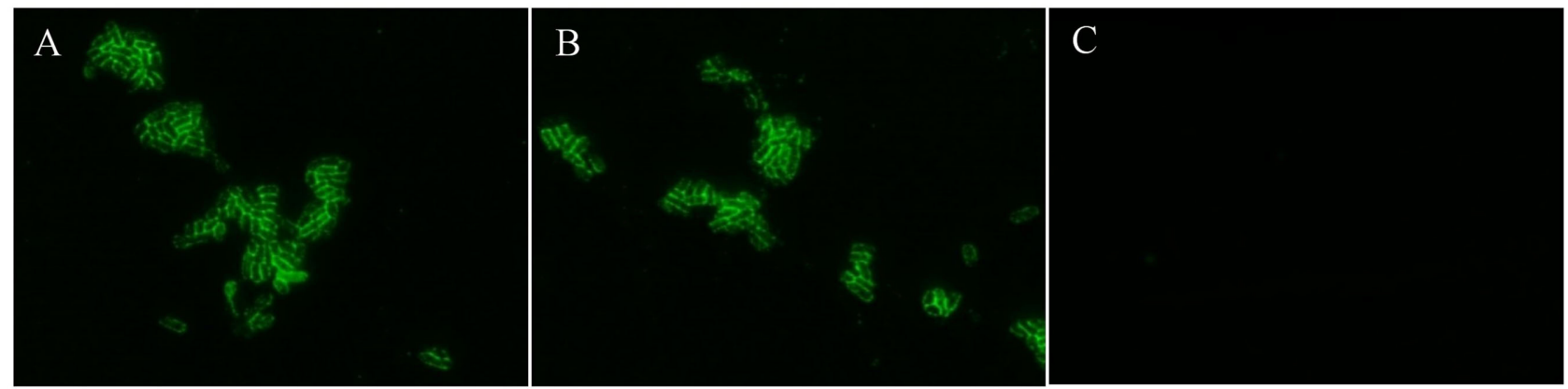

FIGURE 3 | Identification of K88ac expressed on the surface of bacteria by IFA. (A). C83902; (B). E. coli K88ac LT(S63K) $\Delta S T b ;$ (C). E. coli DH5 $\alpha$ as negative control. 


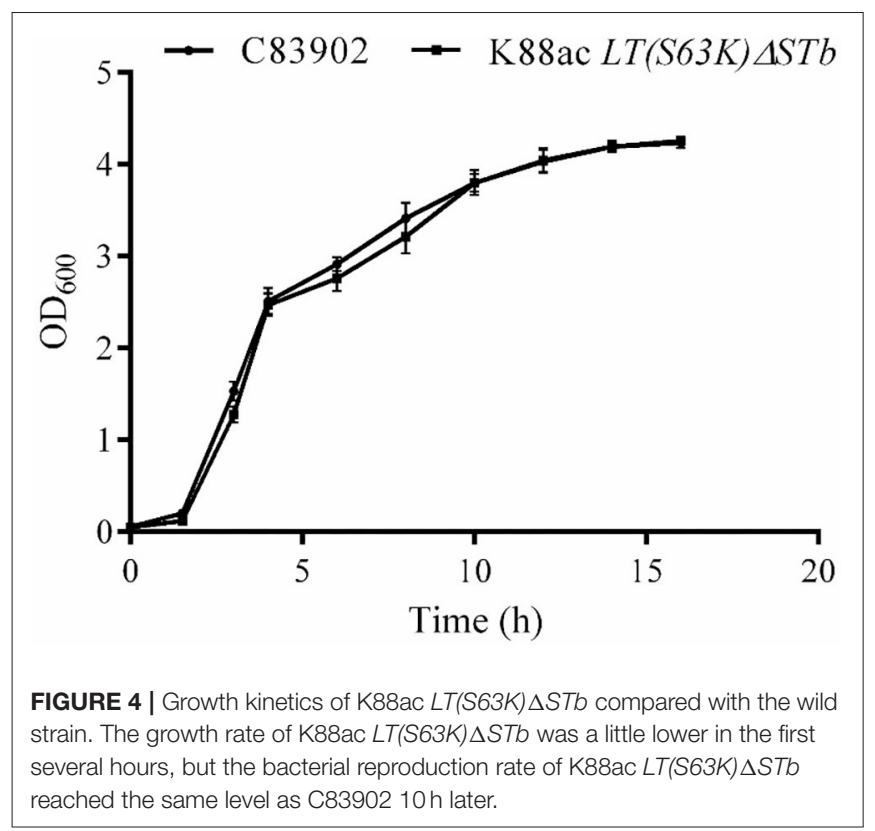

level. This revealed that the attenuated strain had little effect on bacterial reproduction.

\section{K88ac LT(S63K) $\Delta S T b$ Could Not Efficiently Secrete the Active Enterotoxin}

To detect the enterotoxin secretion of the mutated strain E. coli $\mathrm{K} 88 \mathrm{ac} L T(S 63 \mathrm{~K}) \Delta S T$, the enterotoxins were detected using rabbit ligated ileal loop assay. Sterile culture supernatant of K88ac $L T(S 63 K) \Delta S T b$ was injected into rabbit ligated ileal loops. At the same time, the controls of the sterile culture supernatant of E. coli $D H 5 \alpha, \mathrm{C} 83902$, and CAYE medium were also injected into different rabbit ligated ileal loops separately. Twelve hours later, the volumes of collected intestinal liquid in each ligated loop were measured and the ratio of fluid volume to the corresponding ligated loop length was calculated. The results indicated that the average ratio of positive control inoculating culture supernatant of K88ac ETEC (C83902) was 1.27, but others were no more than 0.9 as shown in Figure 5, demonstrating that the mutated strain could not effectively secrete active diarrhea-causing enterotoxin.

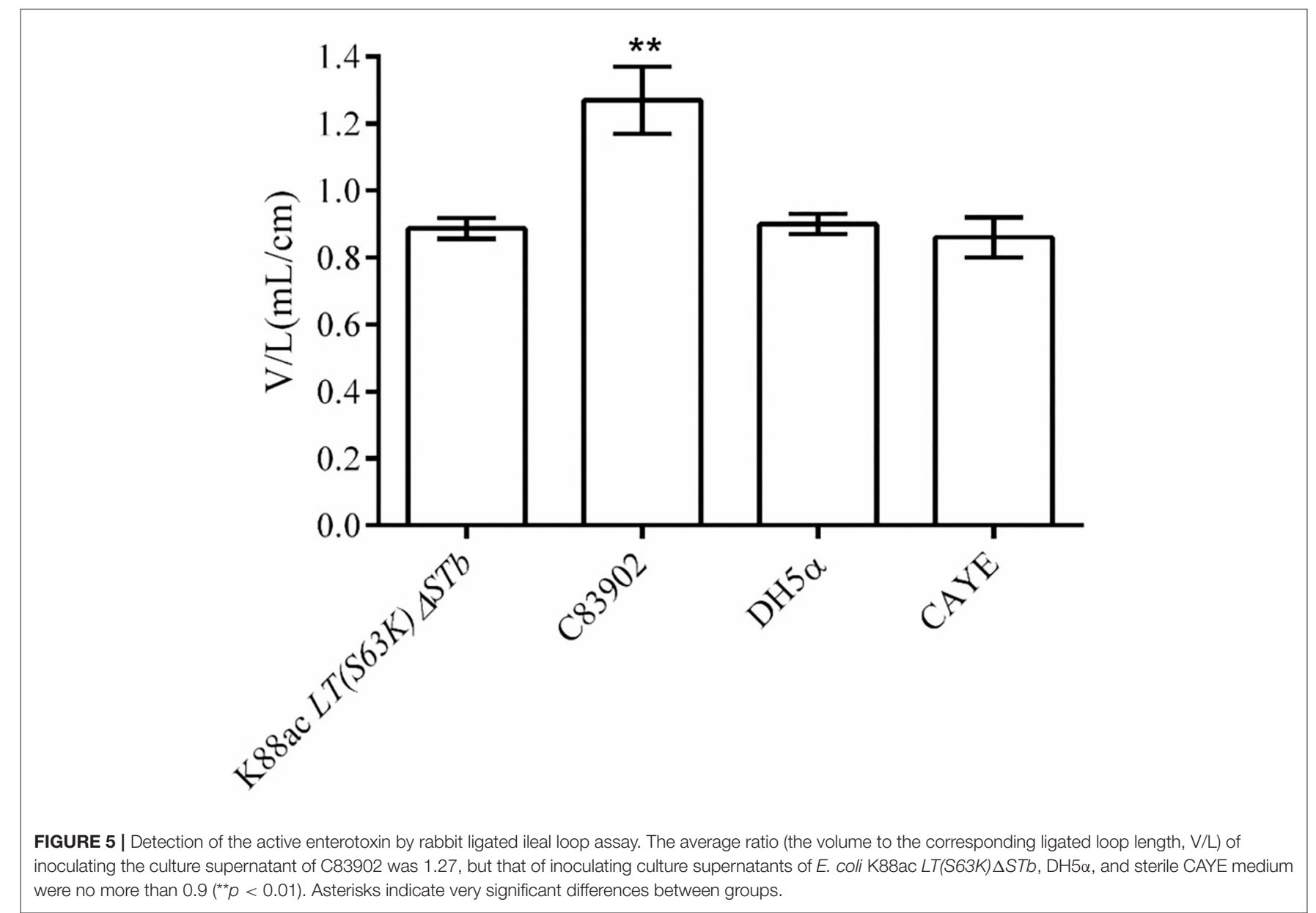




\section{K88ac LT(S63K) $\Delta S T b$ Was Highly Attenuated in Mouse Models and Caused Mucosal Immune Response}

Mice of Group 1 and Group 2 were inoculated with C83902 and K88ac $L T(S 63 K) \triangle S T b$ at doses of $10^{9+} \mathrm{CFU} /$ each, and mice of Group 3 were inoculated with sterile PBS as a control. The clinical scores of each group are recorded in Table 2. In detail, $6 \mathrm{~h}$ later, the clinical symptoms of the mice in Group 1 were as follows: mental depression, slow reactions, ruffled fur and hunchbacked appearances, shivering, dark urine, and diarrhea. Six mice died $12-24 \mathrm{~h}$ post-inoculation, and the remaining mice gradually recovered over the following days. The survival rate of mice in Group 1 was 40\%. Except for mild and transient diarrhea seen in one individual mouse of Group 2, the rest of Group 2 and control Group 3 looked quite normal in subsequent experiments. The survival rates of mice in Group 2 and Group 3 were 100\%. After dissecting the dead mice of Group 1, inflation, emptiness, and yellow effusion in the intestinal tracts were found. The mouse with mild diarrhea in Group 2 was killed by cervical dislocation, and a small amount of fluid was found in the intestinal tract. The intestinal tract of the control mouse in Group 3 was normal, indicating that the virulence of $E$. coli K88ac $L T(S 63 K) \Delta S T b$ to the mouse model was greatly reduced.

Feces were collected separately from the three groups every day. After the enrichment of bacteria, the $L T$ and mutant $L T$ genes in the wild or attenuated strains were detected by PCR to ensure the duration in mouse model. Results indicated that the wild strain could persist for 19 days in mouse intestines, while the attenuated strain could persist for 23 days as shown in Figure 6.

IgA against fimbriae K88ac in feces was detected by indirect ELISA. From the results of indirect ELISA shown in Figure 7, IgA

TABLE 2 | Clinical score recorded after inoculation of E. coli C83902 and K88ac $L T(S 63 K) \Delta S T b$.
DPI

C83092

(Group 1)

56

62

62

62

62

62
K88ac LT(S63K)STb

(Group 2)

3

3

3

3

3

3

3
Control

(Group 3)

0

0

0

0

0

0

0
There were 30 mice that were equally divided into 3 groups and raised in independent units. Mice of each group were inoculated with C83902, K88ac LT(S63K) $\Delta S T$, and sterile $P B S$ as control by intragastric administration at a dose of approximately $10^{9} \mathrm{CFU} /$ each. Clinical symptoms were observed over the following 7 days. Clinical signs were recorded and scored. Clinical symptoms of the mice in Group 1 were exhibited at the 6th hour post-inoculation: 6 mice died, 2 mice had severe diarrhea, and the remaining 2 had mild diarrhea at the end of the first day post-inoculation (DPI); 4 mice had mild diarrhea on the second day, 2 mice were depressed with ruffled fur, and the remaining 2 mice appeared hunchbacked and moved slowly on the third day, then recovered on the following day. Except for mild and transient diarrhea in a single mouse on the first day post-inoculation with K88ac LT(S63K) $\Delta S T$ in Group 2, the mice in Group 2 and control Group 3 looked quite normal during the experimental period. could be detected out as early as the 6th day after inoculation. As time went on, the highest titer of IgA was found on the 12th day, and a high titer of IgA anti-K88ac could still be detected at the 33rd day. This indicated that the attenuated strain could cause a mucosal immune response in the mouse intestinal tract.

\section{K88ac LT(S63K) $\Delta S T b$ Provided Efficient Protection Against the Lethal Challenge}

Mice of Group $1(n=5)$, Group $2(n=5)$, and Group $3(n$ $=40$ ) were treated with fructose, antibiotic, and cimetidine. Then, the mice in Group 1 took C83902 with plasmid pUC18 orally at a dose of $10^{9} \mathrm{CFU} /$ each while the mice in Group 2 and Group 3 took E. coli K88ac $L T(S 63 K) \Delta S T b$ with plasmid pUC18 orally at a dose of $10^{9} \mathrm{CFU} /$ each. Five mice in Group 3 were randomly selected and moved into a new unit at an interval of 24 hours and treated with C83902 containing plasmid pUC18 at a dose of $10^{9} \mathrm{CFU} /$ each until $168 \mathrm{~h}$ post-inoculation with the attenuated bacterium. The last five mice were treated at the $24 \mathrm{~h}$ post-inoculation, when the attenuated bacteria were eliminated from the mouse intestines. Clinical presentations were scored in the following $72 \mathrm{~h}$ post-treatment, and the results indicated that the mice in Group 1 exhibited mental depression, slow reactions, ruffled fur, hunchbacked appearances, and shivering. Four mice died $12-24 \mathrm{~h}$ post-inoculation, and one mouse survived at $72 \mathrm{~h}$ post-inoculation. The mice in Group 2 looked normal in appearance and behavior. In Group 3, all the mice challenged with the original bacterium were normal until the 5th challenge; one mouse exhibited a hunched back and slow reactions in the following $24 \mathrm{~h}$ and recovered the next day at the 6th challenge; one mouse in each unit exhibited mild diarrhea at $24 \mathrm{~h}$, then recovered quickly in the 7 th and 8 th challenge. The clinical scores were recorded in Table 3, and the survival rates of mice in Group 1, Group 2, and Group 3 were 20, 100, and $100 \%$, respectively.

\section{DISCUSSION}

As described previously, our aim was to construct an attenuated strain for an oral whole-cell vaccine against K88ac ETEC based on mutating nucleotides 187-189 of $L T$ from TCT (serine) to AAA (lysine) and deleting $S T b . \lambda$-Red homologous recombination is a common technique used in the mutation and knockout of genes located in chromosomes, but the genes of $L T$ and $S T b$ were in free large plasmids instead of bacterial chromosomes. The unknown copy number of the plasmid, which might lead to incomplete knockout of genes, was the biggest obstacle to using homologous recombination. In this paper, we realized the traceless point mutation in $L T$ and deletion of $S T b$ using two $\lambda$-Red recombination systems and constructed the E. coli K88ac $L T(S 63 K) \Delta S T b$ from wild paternal E. coli C83902 $\left(\mathrm{K} 88 \mathrm{ac}^{+}, \mathrm{LT}^{+}, \mathrm{STb} \mathrm{b}^{+}\right)$, which was verified by PCR and DNA sequencing. The following enterotoxin detection by rabbit ligated ileal loop assay and in vivo test in mice proved that the constructed strain was an attenuated E. coli. 


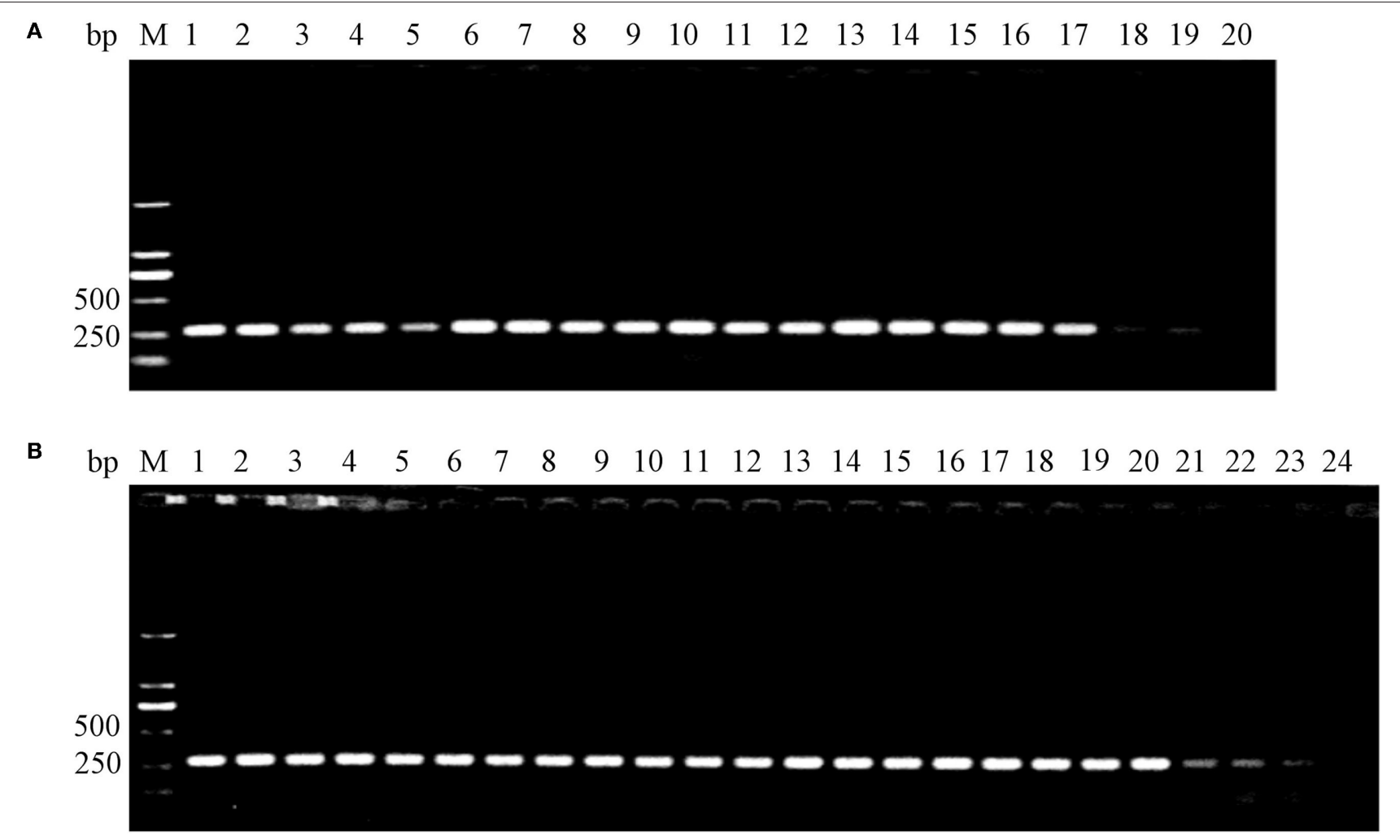

FIGURE 6 | Detection of surviving E. coli K88ac LT(S63K) $\triangle S T$ Tb and C83902 in mouse intestines by PCR. (A). C83902 in feces could survive for 19 days; (B). E. coli $K 88 a c L T(S 63 K) \Delta S T b$ in feces could survive for 23 days.

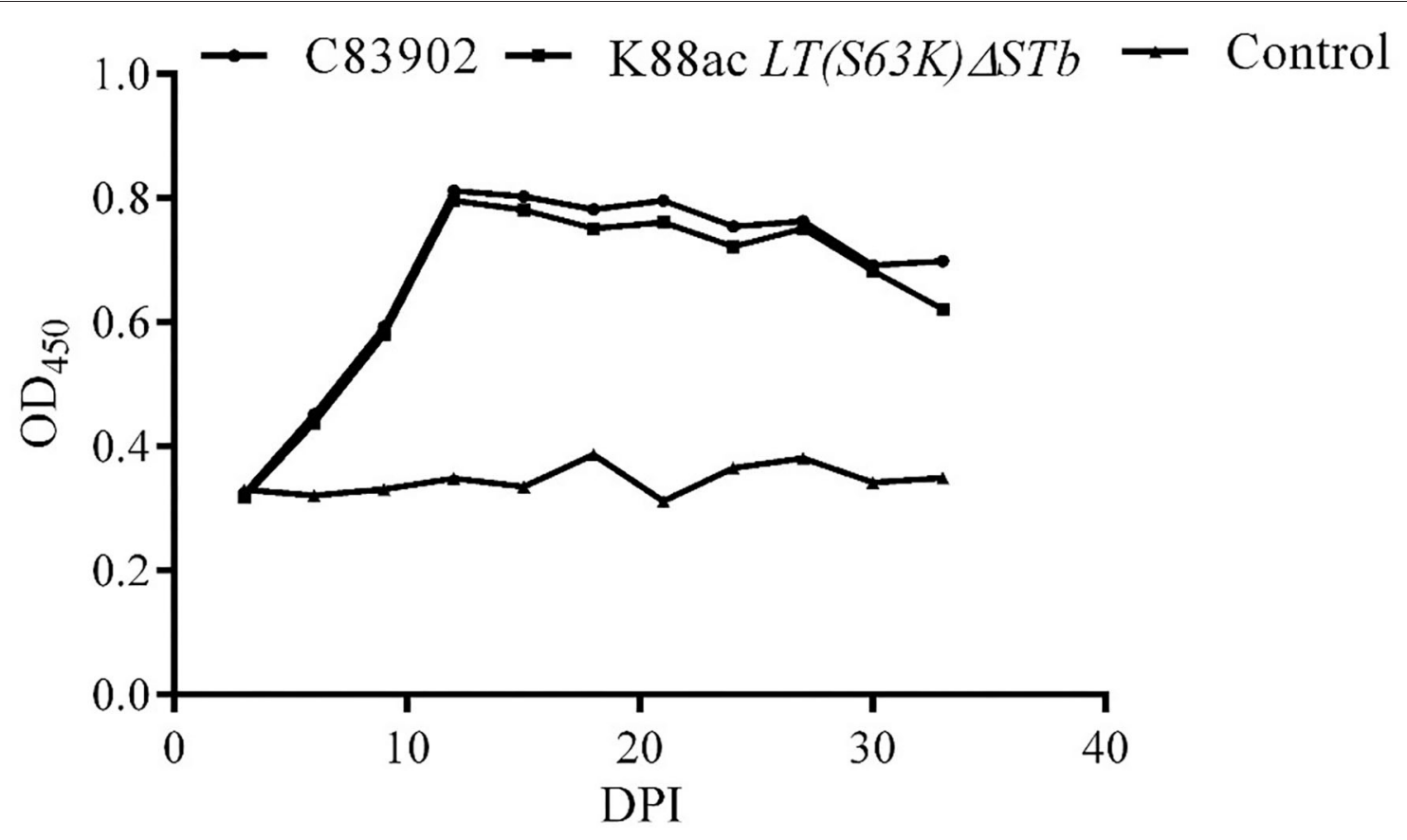

FIGURE 7 | Detection of IgA against K88ac in feces by indirect ELISA. IgA against K88ac could be detected at the earliest on the 6th day post-inoculation (DPI), the highest titer of IgA was found on the 12th day, and high titers of IgA anti-K88ac could still be detected at the 33th day. 
TABLE 3 | Clinical score recorded after inoculation with E. coli C83902, K88ac LT(S63K) $\Delta S T$, and being challenged with C83902.

\begin{tabular}{|c|c|c|c|c|c|c|c|c|c|c|}
\hline DPI & $\begin{array}{c}\text { C83092 } \\
\text { (Group } \\
1 \text { ) }\end{array}$ & $\begin{array}{c}\text { K88ac } \\
L T(S 63 K) S T b \\
\text { (Group 2) }\end{array}$ & \multicolumn{8}{|c|}{ K88ac LT(S63K)STb (Group 3) } \\
\hline 1 & 23 & 0 & & - & - & - & - & - & - & - \\
\hline 2 & 25 & 0 & 0 & - & - & - & - & - & - & - \\
\hline 5 & - & - & - & 0 & 0 & 0 & - & - & - & - \\
\hline 6 & - & - & - & - & 0 & 0 & 0 & - & - & - \\
\hline 7 & - & - & - & - & - & 0 & 0 & 2 & - & - \\
\hline 8 & - & - & - & - & - & - & 0 & 2 & 3 & - \\
\hline 9 & - & - & - & - & - & - & - & 2 & 3 & - \\
\hline 26 & & & & & & & & & & 3 \\
\hline
\end{tabular}

"-" means no recording took place.

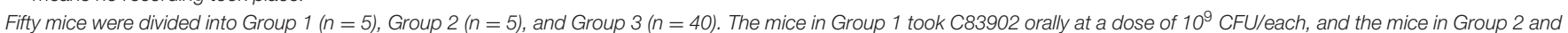

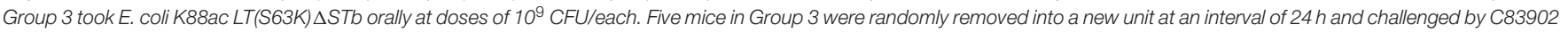

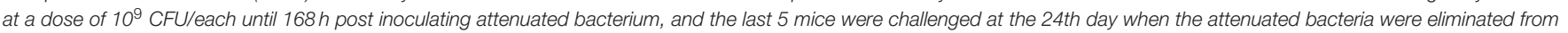

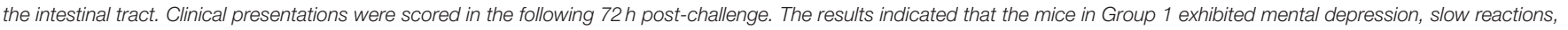

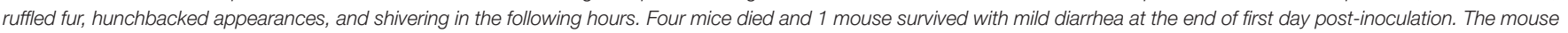

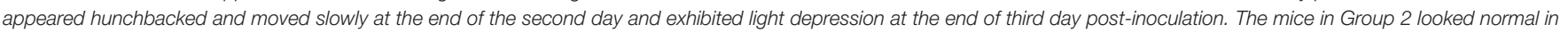

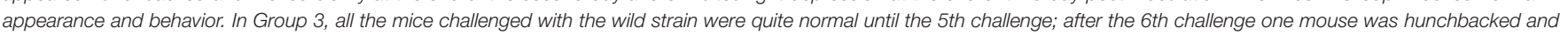

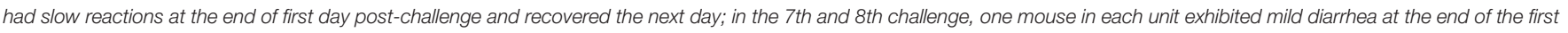
day post challenge, then recovered the next day.

In the process of $\lambda$-Red homologous recombination, imperceptible mistaken deletions may lead to changes in the biological characteristics of bacteria. The characteristics of the mutated bacterium, such as expression of adhesive fimbriae and speed of proliferation, were compared with the original strain. The results demonstrated that the attenuated bacteria expressed the K88ac fimbriae normally, and there was no significant difference in the proliferation rate compared to the wild strain C83902, which provided a reliable theoretical basis for further study of the attenuated strain in animals.

Usually mice are used as animal models in studying pathogenicity and oral ETEC vaccines (9, 20-22). In this paper, the Balb/c mice were inoculated orally with attenuated strains and the pathogenicity of the attenuated strain was significantly decreased compared with the wild parental strain C83902. After taking the attenuated strain, the mice could effectively resist wild K88ac ETEC at doses of $10^{9}$ bacteria in the following 7 days. Usually the first 7 days is very critical for newborn piglets, when K88ac ETEC may result in yellow scour.

IgA is the predominant antibody at the mucosal surface (23). IgA against fimbrial proteins can neutralize the corresponding antigen and inhibit the adhesion of ETEC strains harboring specific receptors present on the brush borders of villous enterocytes (24). The dates showed that the IgA against K88ac secreted by small intestinal mucosa in mice could be detected on the 6th day after inoculation by indirect ELISA. The titer of IgA then gradually increased as time passed and reached the peak at the 12th day, which indicated that the attenuated strain could stimulate mucosal immune response and secrete antibodies against bacteria. However, there was a 5-day blank window period of antibody formation after immunization. Ecological agents such as probiotics can promote the colonization of normal microbiota, increase digestion capacity, reduce intestinal $\mathrm{pH}$ value, and improve mucosal immunity (9). Another important role played by ecological agents is the priority occupying effect, which prevents adhesion and colonization by pathogenic bacteria such as Yersinia enterocolitica and ETEC (25). During the blank window period of IgA formation, the attenuated strain could protect mice against the wild strain C83902, which indicated it might act as a probiotic in the early stage after vaccination. The survival time of the attenuated strain reached 23 days in the intestinal tract of mice, and the results of a challenge at the 24th day postinoculation revealed that the attenuated isolate as a vaccine could effectively protect the mice against wild ETEC. In addition, the attenuated isolate stayed in the intestines for a long time, it might have potential in the development of mucosal immune transport carrier.

In conclusion, this was the first demonstration of the generation of a high attenuated E. coli K88ac $L T(S 63 K) \triangle S T b$ deletion. Escherichia coli K88ac $L T(S 63 K) \Delta S T b$ could elicit mucosal immune response and provide efficient protection against the lethal challenge of ETEC in mouse models, 
highlighting the fact that Escherichia coli K88ac $L T(S 63 K) \Delta S T b$ can be used as a live-attenuated vaccine candidate against ETEC. However, the attenuated phenotype and the protective efficacy of Escherichia coli K88ac $L T(S 63 K) \Delta S T b$ in piglets need to be further evaluated.

\section{DATA AVAILABILITY STATEMENT}

The original contributions presented in the study are included in the article/supplementary materials, further inquiries can be directed to the corresponding author/s.

\section{ETHICS STATEMENT}

The animal study was reviewed and approved by Animal Care and Use Committee of Yangzhou University.

\section{REFERENCES}

1. Fairbrother JM, Nadeau E, Gyles CL. Escherichia coli in postweaning diarrhea in pigs: an update on bacterial types, pathogenesis, prevention strategies. Anim Health Res Rev. (2005) 6:17-39. doi: 10.1079/ahr 2005105

2. Dubreuil JD, Isaacson RE, Schifferli DM. Animal enterotoxigenic Escherichia coli. EcoSal Plus. (2016) 7:1-47. doi: 10.1128/ecosalplus.ESP-0006-2016

3. Zhang W, Zhao M, Ruesch L, Omot A, Francis D. Prevalence of virulence genes in Escherichia coli strains recently isolated from young pigs with diarrhea in the US. Vet Microbiol. (2007) 123:145-52. doi: 10.1016/j.vetmic.2007.02.018

4. Gill DM, Clements JD, Robertson DC, Finkelstein RA. Subunit number and arrangement in Escherichia coli heat-labile enterotoxin. Infect Immun. (1981) 33:677-82. doi: 10.1128/IAI.33.3.677-682.1981

5. Calinescu C, Mulhbacher J, Nadeau E, Fairbrother JM, Mateescu MA. Carboxymethyl high amylose starch (CM-HAS) as excipient for Escherichia coli oral formulations. Eur J Pharm Biopharm. (2005) 60:53-60. doi: 10.1016/j.ejpb.2004.12.006

6. Van den Broeck W, Cox E, Goddeeris BM. Induction of immune responses in pigs following oral administration of purified F4 fimbriae. Vaccine. (1999) 17:2020-9. doi: 10.1016/s0264-410x(98)00406-x

7. Owusu-Asiedu A, Baidoo SK, Nyachoti CM, Marquardt RR. Response of early-weaned pigs to spray-dried porcine or animal plasma-based diets supplemented with egg-yolk antibodies against enterotoxigenic Escherichia coli. J Anim Sci. (2002) 80:2895-903. doi: 10.2527/2002. $80112895 \mathrm{x}$

8. Hill C, Guarner F, Reid G, Gibson GR, Merenstein DJ, Pot B, et al. Expert consensus document. The International Scientific Association for Probiotics and Prebiotics consensus statement on the scope and appropriate use of the term probiotic. Nat Rev Gastroenterol Hepatol. (2014) 11:506-14. doi: 10.1038/nrgastro. 2014.66

9. Uyeno Y, Shigemori S, Shimosato T. Effect of probiotics/prebiotics on cattle health and productivity. Microbes Environ. (2015) 30:126-32. doi: $10.1264 /$ jsme2.ME14176

10. Wen LJ, Hou XL, Wang GH, Yu LY, Wei XM, Liu JK, et al. Immunization with recombinant Lactobacillus casei strains producing K99, K88 fimbrial protein protects mice against enterotoxigenic Escherichia coli. Vaccine. (2012) 30:3339-49. doi: 10.1016/j.vaccine. 2011.08.036

11. Choi C, Chae C. Genotypic prevalence of F4 variants (ab, ac, and ad) in Escherichia coli isolated from diarrheic piglets in Korea. Vet Microbiol. (1999) 67:307-10. doi: 10.1016/s0378-1135(99) 00046-2

\section{AUTHOR CONTRIBUTIONS}

XZ and SY completed most of the work. YY helped with rabbit surgery. DC, YF, HS, and JD gave some beneficial advice. FW designed most of the study. All authors contributed to the article and approved the submitted version.

\section{FUNDING}

This research was funded by the Priority Academic Program Development (PAPD) of Jiangsu Higher Education Institutions.

\section{ACKNOWLEDGMENTS}

We thank all of the authors for their contributions to the study.

12. Ochi S, Shimizu T, Ohtani K, Ichinose Y, Arimitsu H, Tsukamoto K, et al. Nucleotide sequence analysis of the enterotoxigenic Escherichia coli Ent plasmid. DNA Res. (2009) 16:299-309. doi: 10.1093/dnares/ dsp015

13. Akker FVD, Pizza M, Rappuoli R, Hol WGJ. Crystal structure of a non-toxic mutant of heat-labile enterotoxin, which is a potent mucosal adjuvant. Protein Sci. (2010) 6:2650-4. doi: 10.1002/pro.55 60061220

14. Horstman AL, Bauman SJ, Kuehn MJ. Lipopolysaccharide 3-deoxyD-manno-octulosonic acid (Kdo) core determines bacterial association of secreted toxins. J Biol Chem. (2004) 279:8070-5. doi: 10.1074/jbc. M308633200

15. O'Neal CJ, Jobling MG, Holmes RK, Hol WG. Structural basis for the activation of cholera toxin by human ARF6-GTP. Science. (2005) 309:1093-6. doi: 10.1126/science.1113398

16. Pizza M, Domenighini M, Hol W, Giannelli V, Fontana MR, Giuliani MM, et al. Probing the structure-activity relationship of Escherichia coli LT-A by site-directed mutagenesis. Mol Microbiol. (1994) 14:51-60. doi: 10.1111/j.1365-2958.1994. tb01266.x

17. Hu FX, Ding R, Cui ZH, Yu JL, Liu LF, Ao YH, et al. Approaches and strategies of gene scarless knockout in the Escherichia coli genome. Letters Biotechnol. (2013) 24:552-7. doi: 10.3969/j.issn.1009-0002. 2013.04.026

18. Syngkon A, Elluri S, Koley H, Rompikuntal PK, Saha DR, Chakrabarti $\mathrm{MK}$, et al. Studies on a novel serine protease of a DeltahapADeltaprtV Vibrio cholerae O1 strain and its role in hemorrhagic response in the rabbit ileal loop model. PLoS ONE. (2010) 5:e13122. doi: 10.1371/journal.pone. 0013122

19. Kolpe AB, Kiener TK, Grotenbreg GM, Kwang J. Display of enterovirus 71 VP1 on baculovirus as a type II transmembrane protein elicits protective $\mathrm{B}$ and $\mathrm{T}$ cell responses in immunized mice. Virus Res. (2012) 168:64-72. doi: 10.1016/j.virusres.2012.06.014

20. Laird RM, Ma Z, Dorabawila N, Pequegnat B, Omari E, Liu Y, et al. Evaluation of a conjugate vaccine platform against enterotoxigenic Escherichia coli (ETEC), Campylobacter jejuni and Shigella. Vaccine. (2018) 36:6695-702. doi: 10.1016/j.vaccine.2018.09.052

21. Ran X, Chen X, Wang S, Chang C, Wen X, Zhai J, et al. Preparation of porcine enterotoxigenic Escherichia coli (ETEC) ghosts and immunogenic analysis in a mouse model. Microb Pathog. (2019) 126:224-30. doi: 10.1016/j.micpath. 2018.11.015

22. Rodea GE, Montiel-Infante FX, Cruz-Cordova A, Saldana-Ahuactzi Z, Ochoa SA, Espinosa-Mazariego K, et al. Tracking bioluminescent ETEC during in vivo BALB/c mouse colonization. Front Cell Infect Microbiol. (2017) 7:187. doi: 10.3389/fcimb.2017.00187 
23. Virdi V, Coddens A, De Buck S, Millet S, Goddeeris BM, Cox E, et al. Orally fed seeds producing designer IgAs protect weaned piglets against enterotoxigenic Escherichia coli infection. Proc Natl Acad Sci USA. (2013) 110:11809-14. doi: 10.1073/pnas. 1301975110

24. de Oliveira IR, Bessler HC, Bao SN, Lima RD, Giugliano LG. Inhibition of enterotoxigenic Escherichia coli (ETEC) adhesion to Caco-2 cells by human milk and its immunoglobulin and non-immunoglobulin fractions. Braz J Microbiol. (2007) 38:86-92. doi: 10.1590/S1517-838220070 00100018

25. Yu X, Avall-Jaaskelainen S, Koort J, Lindholm A, Rintahaka J, von Ossowski I, et al. A comparative characterization of different host-sourced lactobacillus ruminis strains and their adhesive, inhibitory, immunomodulating functions. Front Microbiol. (2017) 8:657. doi: 10.3389/fmicb. 2017.00657
Conflict of Interest: The authors declare that the research was conducted in the absence of any commercial or financial relationships that could be construed as a potential conflict of interest.

The reviewer GZ declared a shared affiliation with several of the authors, $\mathrm{XZ}$, SY, DC, YY, HS, to the handling editor at time of review.

Copyright (c) 2021 Zhang, Yu, Cheng, Feng, Yang, Sun, Ding and Wang. This is an open-access article distributed under the terms of the Creative Commons Attribution License (CC BY). The use, distribution or reproduction in other forums is permitted, provided the original author(s) and the copyright owner(s) are credited and that the original publication in this journal is cited, in accordance with accepted academic practice. No use, distribution or reproduction is permitted which does not comply with these terms. 